\title{
Physical Ecology: the Search for Life Law
}

\author{
Josep Penuelas $^{1,2, *}$ and Jaume Terradas ${ }^{1,3}$ \\ ${ }^{I}$ CREAF, Edifici C, Universitat Autònoma de Barcelona Cerdanyola del Vallès, Barcelona 08193, Catalonia, Spain \\ ${ }^{2}$ CSIC, Global Ecology Unit CREAF-CEAB-CSIC-UAB, Cerdanyola del Vallès, Barcelona 08193, Catalonia, Spain \\ ${ }^{3}$ Department of Plant and Animal Biology and Ecology of the Universitat Autònoma de Barcelona, Cerdanyola del \\ Vallès, Barcelona 08193, Catalonia, Spain
}

Abstract: Life on Earth is the result of a continuous accumulation of information by combination and innovation riding on endo and exosomatic energy gradients and discontinuous destructions.

Keywords: Physical ecology, information, combination, innovation, endosomatic energy, exosomatic energy, discontinuous destructions, energy flow, culture, social systems.

Earth life is complex and diverse. It is made of discontinuous individuals belonging to millions of different species that overlap materially or in their influences in the available space, with the events in that milieu in turn contributing back to life. This complexity and diversity of life deployment is ruled by few processes and physical laws. In fact, three of these processes, combination, innovation and death, modulate life in Earth while it is riding on a thermodynamic gradient of endo and exosomatic energy use with continuous accumulation of information and discontinuous destructions.

The more complex and diverse a system is, the more information it contains. This occurs in ecological systems as well as in physical or cultural ones. In the fifties and sixties of the last century, ecology was more interested in energy and matter flows and budgets, but some researchers, like Margalef (1997), enhanced the importance of the third Aristotelian principle, the form, reinterpreted as information. To understand how information accumulates in organisms and ecosystems is the main challenge of biology and ecology. The process of combination builds atoms from particles, or molecules from atoms, and permits genetic transfer between species, integration of symbionts and other processes that provide species new tool boxes, and thus, new abilities (Terradas \& Penuelas, 2009). So, pre-existing pieces (in the environment or other organisms) are assembled in bigger structures. Living organisms store and cheaply copy information, and the copied pieces are modified by mutation and other genetic mechanisms in an additional process of innovation. The same process occurs in culture. In that way, both in biology and culture two processes create variety: combination (called incorporation when one piece is much larger that the other one) and innovation. Complexity and

*Address correspondence to this author at the CREAF, Edifici C, Universitat Autònoma de Barcelona Cerdanyola del Vallès, Barcelona 08193, Catalonia, Spain; Tel 34935811312; Fax: 34935814151;

E-mail: josep.penuelas@uab.cat diversity, both in life and culture, result from combination and innovation. Enormous complexity and diversity can be attained by assembling in different ways a relatively small number of elements, as it occurs in language or chemistry: around 25 letters or around 100 chemical elements can combine to form ensembles that, ordered or interacting in different ways, produce unimaginable numbers of possibilities. By assembling pieces, we can build gothic churches or spaceships.

Life depends on the energy flow to sustain metabolism: plants use solar energy, animals and fungi use organic matter, and bacteria and archaea can use a variety of energy chemical sources. Ecosystems consist of very complex networks of interacting species (at different levels of space and time) and a physicochemical environment. These networks are hierarchical, organized under the flow of energy, in a stepping process, while it is dissipated to heat. In fact, because of this link between life and energy dissipation, life can be seen as a manifestation of the second law of thermodynamics (Schneider and Kay, 1994). But jointly with this relatively feeble endosomatic flow of energy that permits metabolism, organisms and ecosystems depend in many ways on high amounts of exosomatic energy (Margalef 1997). Evapotranspiration rises water and nutrients from soil to the leaves in plants. Hence this transport is possible thanks to solar radiation. Also evaporation and solar radiation are responsible for ventilation and climate regulation in termite and ants buildings. Many species benefit from wind or water transport to disperse, hunting or finding reproductive partners. Some of them are especially effective in using at their own benefit exosomatic energies. In the case of mankind, that trend has been much promoted by the advantage of cultural transmission of knowledge and fast communication to the human population. The enormous cultural development of the use of exosomatic energy by mankind is an extreme case of a more general trend in life evolution. It allows a continuously increasing use and control of space and resources that have been never exploited before, and also feed backs cultural evolution and population 
growth, but it also involves an increasing danger of disturbance of climate, ocean circulation, biodiversity loss and resources exhaustion.

This effectiveness in using exosomatic energies is promoted by evolutionary traits such as social organization with labour division and handling abilities, and brain capacities for both. This effectiveness in using exosomatic energies has multiple implications in human society. For example, organizational constraints arising from energy gain predict changes to settlement and organization in postcarbon societies (Tainter et al., 2003). Physicist and economist John L.R. Proops related the exosomatic energy consumption of the services and government sector with the increasing interconnectedness between individuals and the resource flows used by a society. But social systems continue to function on the same principles: combinationincorporation and innovation: most insect societies integrate other species, as fungi or bacteria, in their organization (Hölldobler and Wilson, 2009) and human societies incorporate domesticated plants and animals, pets, managed forests and grasslands, artefacts, even robots. This occurs for social systems in a similar way to that occurring in individual organisms, where large animal (including human) bodies contain many millions of microbes that are essential because they contribute to digestion and other processes, and humans even incorporate increasingly sophisticated prosthesis.

Culture is a natural product of evolution in animal societies. It has been defined (by Kinji Imanishi in the fifties, cited by Frans de Waals, 2001) as non-genetic transmission of behaviour. With this definition, a monist idea of evolution is easily reached, breaking down the ideological barriers of the dualism Man-Nature. The existence of specific behaviours developed by innovation and culturally reproduced inside a local group of individuals has been observed in many kinds of animals, including some birds, many terrestrial and marine mammals and, obviously in primates. These cultural developments permit interesting responses to diverse aspects of local environments, and sometimes an increase of the share of energy flow driven to the species benefit. Symbolic thinking has been discovered apparently only in man and in extinct Homo species, but consciousness, self-consciousness and social transmission of non-inherited behaviour are much more general. And then, culture is a logical consequence of biological evolution. We need to include mankind and culture in an overarching evolutionary and ecological theory, if we want to integrate ecology and socio-economics and to change the dominant view of Earth and Biosphere as sources of resources that we can use in our benefit by an increasing use of energy and the correlated consequences of destabilization of life-supporting systems. A new physical biology and ecology can give us a global understanding of our dependence on those systems and the tools for mankind behaviour on Earth becoming more adaptive than exploitative.

The process of acquisition of information is historical and can not be run in reverse, it can not be played back to disentangle each block of added information in an inverse valid way. Asymmetry rules life in relation to individual time birth, growth, and death, ecosystems succession and its catastrophic destruction, cultures deployment and collapses: there is an hysteresis, construction is slow (energy is piled gradually in the system), whereas destruction can be very fast (energy is then liberated suddenly). Changes towards simplification occur discontinuously, apparently distributed at random, and each one erases large partial stores of the accumulated information, but it is impossible to extract orderly one after the other, the pieces that came with successive inputs and interactions in biological evolution. The most efficient and cheapest solution, since the mechanisms of genetic copying and reproduction of the organisms work so well, and are thermodynamically cheap, is to include death as a normal event in the program. This occurs also at the level of ecosystems, although in that case with components that are less integrated, making some manipulation and restoration possible: ecosystems are cyclically destroyed by fire, wind or other disturbances. The entire planet Earth has probably passed periods of nearly complete destruction of previous ecosystems (snow-ball Earth, oxygen catastrophe, etc.). It may even be the case at the universe level. Why not include the Big Bang also in this kind of events? A bang big enough could sweep clean the blackboard of a particular Universe. Reset and start again (Margalef, 1997). It does not matter if this reset is due to a cyclical process of expansion-collapse of a unique Universe (Penrose, 2010) or occurs in individual Universes from a multi-verse.

All life and ecological processes are built in a thermodynamic continuum where entropies add and information multiplies. As a result, it is more effective (the winner strategy in a selective game) to join to an existing store than to start a new one. Information accumulates easily and faster when there is already a large core of it. Its growth is allometric, as most processes concerning life are. After a large collapse (i.e., a wildfire) the system is re-built in a large part from remaining pieces. In Earth history, after the great extinctions, space and resources are conquered by survivors that evolve and diversify (adaptive radiations) fast to form a new hierarchical network of energy paths: after dinosaurs became extinct, mammals diversified from survivor species and filled the gap. Saint Matthew's principle appears here stating that those who already have more get also more due to the advantage of previous organization. In dealing with information, a brain twice larger than another is more than twice as powerful. Hence, time offers the possibility to life systems to enlarge and complicate structures, whereas keeping them functionally coherent, up to a limit imposed by the distance between potential reactants. In that way, more complex systems may appear as time flows and history accumulates. This is how life on Earth generates its huge complexity, a complexity and heterogeneity that can be seen as a noise in physics but that we, ecologists, see as the magic of life.

\section{CONFLICT OF INTEREST}

The authors confirm that this article content has no conflicts of interest.

\section{ACKNOWLEDGEMENTS}

The authors' research is funded by Spanish Government Grants CGL2010-17172/BOS and Consolider-Ingenio Montes Grant CSD2008-00040 and by Catalan Government Grant SGR 2009-458 and SGR 2009-1511. 


\section{REFERENCES}

Hölldobler, B., and Wilson, E.O. (2009). The Superorganism: The Beauty, Elegance, and Strangeness of Insect Societies, USA: W.W. Norton \& Company, Inc.

Margalef, R. (1997). Our Biosphere. Excellence in Ecology series. Germany: International Ecology Institute.,.

Penrose, R. (2010). Cycles of Time: An Extraordinary New View of the Universe. Bodley Head London.

Proops, J.L (1979). Energy, Entropy and Economic Structure. UK: University of Keele. p. 506.
Schneider, E.D. and Kay, JJ (1994). Life as a manifestation of the second law of thermodynamics. Mathematical and Computer Modelling 19, 25-48.

Tainter, J.A., Allen T.F.H, Little A, \& Hoekstra T.W. (2003). Resource transitions and energy gain: contexts of organization. Conservation Ecology 7(3), 4. Available from: http://www.consecol.org/ vol. 7, no. 3 , art 4

Terradas, J. \& Penuelas, J. (2009). Evolution: Much More than Genetics. the need for a holistic view. The Open Evolution Journal, 3, 38-45.

Waals, F. D. (2001). The Ape and the Sushi Master, Cultural reflections by a primatologist. New York: Basic Books.

Received: February 15, 2013

Revised: April 04, 2013

Accepted: April 15, 2013

(C) Penuelas and Terradas; Licensee Bentham Open.

This is an open access article licensed under the terms of the Creative Commons Attribution Non-Commercial License (http://creativecommons.org/ licenses/by-nc/3.0/), which permits unrestricted, non-commercial use, distribution and reproduction in any medium, provided the work is properly cited. 